\title{
Cross-check of ex-situ and in-situ metrology of a bendable temperature stabilized $\mathrm{KB}$ mirror
}

\author{
Sheng Yuan, ${ }^{\text {a, }}$ Kenneth A. Goldberg, ${ }^{\mathrm{b}}$ Valeriy V. Yashchuk, ${ }^{\mathrm{a}}$ Richard Celestre, ${ }^{\mathrm{a}}$ \\ Wayne R. McKinney, ${ }^{\mathrm{a}}$ Gregory Morrison, ${ }^{\mathrm{a}}$ James Macdougall, ${ }^{\mathrm{b}}$ Iacopo Mochi, ${ }^{\mathrm{b}}$ Tony Warwick ${ }^{\mathrm{a}}$ \\ ${ }^{a}$ Advanced Light Source, Lawrence Berkeley National Laboratory, Berkeley, CA 94720, USA \\ ${ }^{b}$ Center for X-ray Optics, Lawrence Berkeley National Laboratory, Berkeley, CA 94720, USA \\ * E-mail: syuan@lbl.gov; Phone: +1-510-495-2592; Fax: +1-510-486-7696
}

\begin{abstract}
At the Advanced Light Source (ALS), we are developing broadly applicable, high-accuracy, in-situ, atwavelength wavefront slope measurement techniques for Kirkpatrick-Baez (KB) mirror nano-focusing. In this paper, we report an initial cross-check of ex-situ and in-situ metrology of a bendable temperature stabilized KB mirror. This cross-check provides a validation of the in-situ shearing interferometry currently under development at the ALS.
\end{abstract}

Keywords: x-ray, synchrotron radiation, free electron laser, KB mirror, metrology, wavefront measurements, shearing interferometry, optical slope metrology, long trace profiler

\section{Introduction}

Nano-focusing and coherence preservation for third-generation synchrotron radiation and free electron laser beamlines requires tight reflective x-ray optics surface slope tolerances. While the fabrication quality and ex-situ metrology of x-ray mirrors has improved over time [1-3], the insitu, at-wavelength performance of beamline optics is often limited by application specific factors, such as beamline optics alignment, temperature and mechanical drift, vibration, etc. [4-8].

At the Advanced Light Source (ALS), we are developing broadly applicable, high-accuracy, insitu, at-wavelength wavefront metrology techniques, suitable for high precision tuning, alignment, and performance characterization of Kirkpatrick-Baez (KB) mirrors [9-11]. The methodology of the work relies on development of a series of at-wavelength metrology techniques with sequentially increasing accuracy and sensitivity. The efficacy of the techniques is verified in experiments at the ALS beamline 5.3.1 with a single, bendable, temperature stabilized KB mirror [8] designed for sub-micrometer focusing of soft x-rays near 1-nm wavelength [12]. Use of the same test mirror and the same beamline arrangement allows us to reliably cross-check various at-wavelength metrology techniques and compare their performance with that of the optical metrology available at the ALS optical metrology laboratory (OML) [3,13-19].

In this paper, we report the first results on development at the ALS of a soft x-ray shearing interferometry technique. The technique is applied to optimally align and characterize a KB mirror pre-bent into the correct shape at the ALS optical metrology laboratory (OML). The mirror surface slope profile, extracted from the in-situ measurements, is compared with the result of optical surface slope metrology performed with a long trace profiler, the ALS LTP-II [15]. After recent upgrade [15], the ALS LTP-II is capable for surface slope measurements with accuracy of about $0.1 \mu \mathrm{rad}$ (rms) with flat and slightly curved optics and less than $0.25 \mu \mathrm{rad}$ (rms) with significantly curved x-ray optics. Therefore, a cross-check with the high performance 
optical instrument provides a reliable validation of the in-situ shearing interferometry technique currently under development at the ALS.

\section{Beamline arrangement}

Metrology beamline 5.3.1 at the ALS is a bend-magnet beamline that provides an intense x-ray beam in a broad energy range from $30 \mathrm{eV}$ to $12 \mathrm{keV}$. Figure 1 shows the beamline arrangement. $\mathrm{X}$-rays from a bending magnet are focused by a 1:1 toroidal mirror, M1, and pass through a monochromator. The monochromator comprises a pair of $\mathrm{W} / \mathrm{B}_{4} \mathrm{C}$ multilayer mirrors with a $4 \mathrm{~nm}$ period. The M1 toroidal mirror focuses the incident x-rays 12-m downstream, inside a 2-m-long vacuum chamber of a dedicated experimental endstation. The focal spot was measured to have a FWHM size of $150 \mu \mathrm{m}$ (vertical) $\times 300 \mu \mathrm{m}$ (horizontal). The details of the endstation design have been described elsewhere [11].

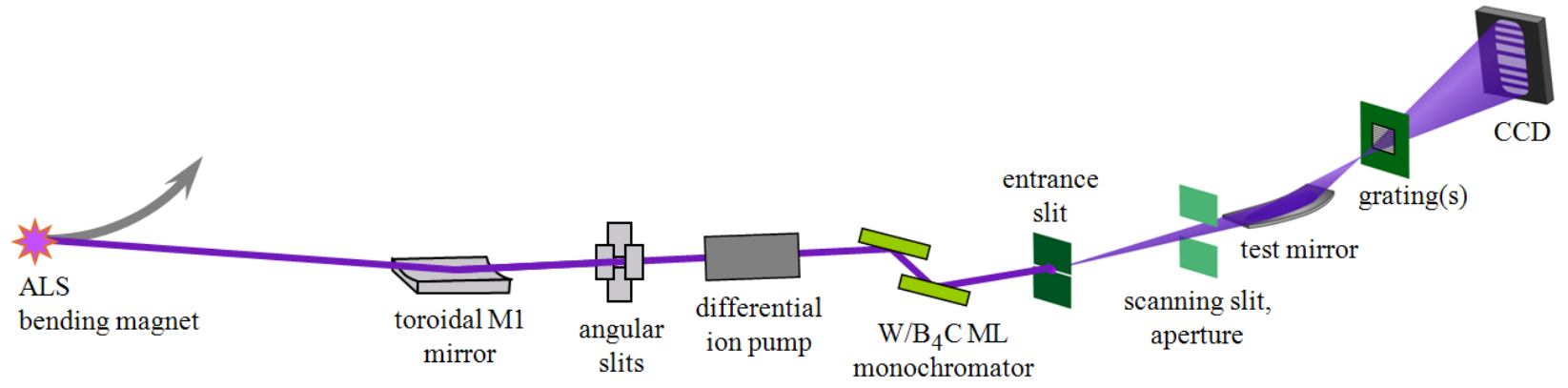

Figure 1: Beamline 5.3.1 arrangement

In order to generate a cylindrical reference beam for one-dimensional focusing tests, we use a nano-fabricated entrance slit spatial filter. The filter, designed as an array of 200 - $\mu \mathrm{m}$-long slits with widths between 0.66 and $6.00 \mu \mathrm{m}$, was fabricated by electron-beam lithography. The multislit design enables the optimal selection of the slit size in-situ. The filter is placed in the focus of M1 mirror, a position that coincides with the object plane of the KB mirror.

\section{Temperature stabilized test mirror}

Figure 2 shows details of the test mirror design. The same mirror was used in our previous work [11]. The bending mechanism of the mirror is based on two cantilever springs. With a wire, each cantilever spring is connected to a displacement-reduction spring that is driven with a Picomotor $^{\mathrm{TM}}$. The displacement of the Picomotor ${ }^{\mathrm{TM}}$ actuators is monitored with linear variable differential transformers (LVDT) with an accuracy of approximately $100 \mathrm{~nm}$ over the useful range. The bender design allows extremely fine control of the bending couples applied to the mirror substrate.

The mirror design and the selection of the materials used for its fabrication allow efficient temperature stabilization of the mirror by a Peltier element attached directly to body of the mirror assembly. The efficiency of the temperature stabilization has been investigated in Ref. 8 . We have demonstrated that a change of the ambient temperature by a few degrees $\mathrm{C}$ does not noticeably affect the mirror shape.

The mirror substrate is made of crystal silicon and has 102-mm length and 4-mm thickness. The substrate is side-profiled to enable it to achieve the desired elliptical shape when optimally bent. 


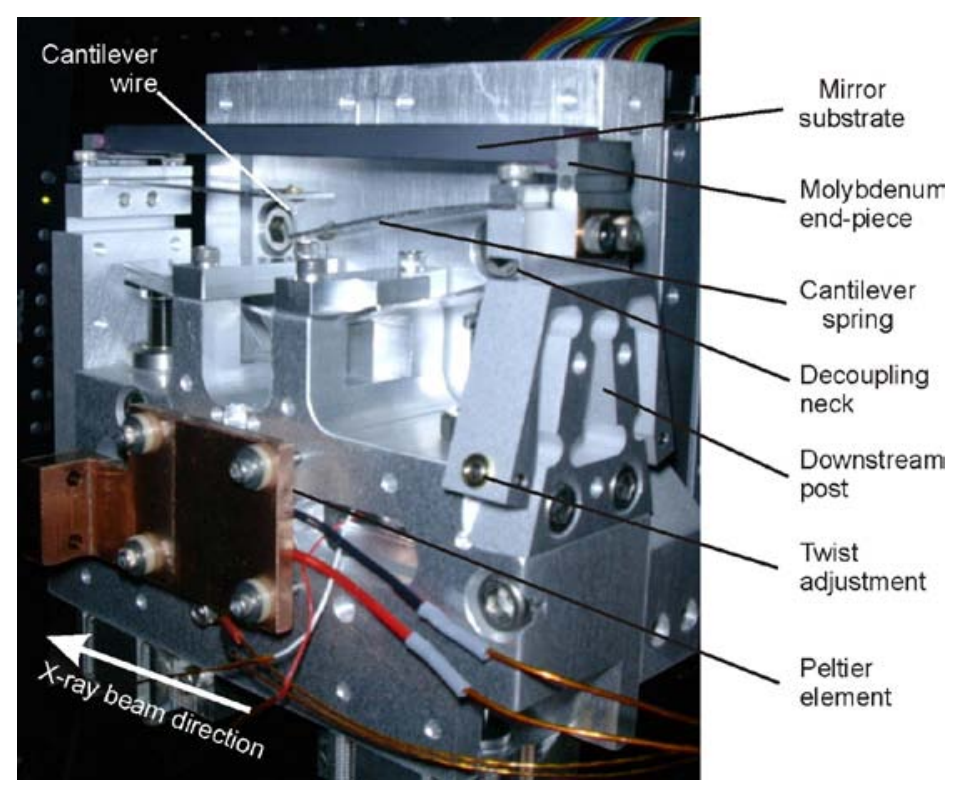

Figure 2: Bendable, temperature-stabilized test mirror on the KB bender. Here the mirror's reflective surface faces upward.

The assembly, preliminary alignment, and the setting of the mirror benders are performed by monitoring the mirror surface shape with a 6-inch $\mathrm{ZYGO}^{\mathrm{TM}}$ GPI interferometer at the OML. The twist in the mirror substrate is removed using the dedicated upstream anti-twist adjustment, shown in Fig. 2. First, the anti-twist correction is performed with the interferometer, observing the entire clear aperture of the mirror. Second, for more precise correction, we use the Developmental LTP (DLTP) [3] to measure and minimize the slope variation in the sagittal direction when scanning along the tangential axis. With this step, the mirror shape becomes close to the desired elliptical surface.

\section{The ex-situ LTP measurement of the test mirror}

After the preliminary alignment, and before installation at the beamline, the test mirror benders are optimally set for the desired mirror shape and precisely characterized with the upgraded ALS LTP-II [15].

Table 1 presents the mirror's optical specifications for the present experiments. In Table 1 , the radius of curvature is given for the mirror center.

Note that this mirror was originally designed with a shape optimized for a smaller grazing incidence angle $\theta=4 \mathrm{mrad}$ and therefore, a smaller numerical aperture $N A \approx 1.5 \mathrm{mrad}$. At 1-nm wavelength (approximately $1 \mathrm{keV}$ ), diffraction-limited focusing with the originally specified shape would provide a focal spot with a size of about $0.2 \mu \mathrm{m}$. Our goal was to achieve a smaller focal spot using a larger $N A$, at $\sim 3$ mard.

In order to increase mirror NA thus decrease the diffraction limited focal spot size, the mirror is bent to a new set of parameters, as listed in Table 1, selected from numerical mirror bending simulation $[9,20]$. 
Table 1: Optical specifications of the test mirror.

\begin{tabular}{|c|c|c|c|c|}
$\begin{array}{c}\text { object } \\
\text { distance, } r\end{array}$ & $\begin{array}{c}\text { image } \\
\text { distance, } r^{\prime}\end{array}$ & $\begin{array}{c}\text { grazing } \\
\text { angle, } \theta\end{array}$ & $\begin{array}{c}\text { numerical } \\
\text { aperture, NA }\end{array}$ & $\begin{array}{c}\text { radius of } \\
\text { curvature, } R_{0}\end{array}$ \\
\hline $1600 \mathrm{~mm}$ & $120 \mathrm{~mm}$ & $8.0 \mathrm{mrad}$ & $3 \mathrm{mrad}$ & $27.91 \mathrm{~m}$
\end{tabular}

To optimize the bender setting, we use an original procedure for the tuning and calibration of bendable mirrors with slope measuring profilers [13,14], developed in our laboratory. The procedure consists of three slope profile measurements with the sequential change of the two bender settings. The observed shape changes allow us to isolate the contributions from each bender when the system is close to its optimized bending state. Based on the measurements, predictions for the optimal settings and their confidence intervals are calculated using linear regression analysis (see e.g., Ref. 19 and references therein). The optimization procedure removes defocus and coma aberrations (2nd and 3rd order shape errors) at the design conjugate distances. After a couple of bender setting iterations, the mirror shape was adjusted to the desired ellipse, given by the parameters in Table 1 , with an rms error of about $0.4 \mu \mathrm{rad}$.

Figure 3 shows a trace of the residual slope error over an $80-\mathrm{mm}$ clear aperture of the optimally bent test mirror. The trace, with a uniform sampling along the mirror clear aperture with a 1-mm increment, is a result of subtraction of the desired slope profile from the measured mirror surface slope trace. In order to suppress the contributions of the instrumental systematic and drift errors, LTP measurements were performed according to an optimal scanning strategy described in Ref. 18. In our case, the measurement run consisted of eight consecutive measurements performed with scanning the LTP optical sensor in the forward (F) and the back (B) direction according to a sequence of F-B-B-F-B-F-F-B. The chosen scanning strategy allows for effective suppression of an error due to the instrumental drift described with a third order polynomial function of time.

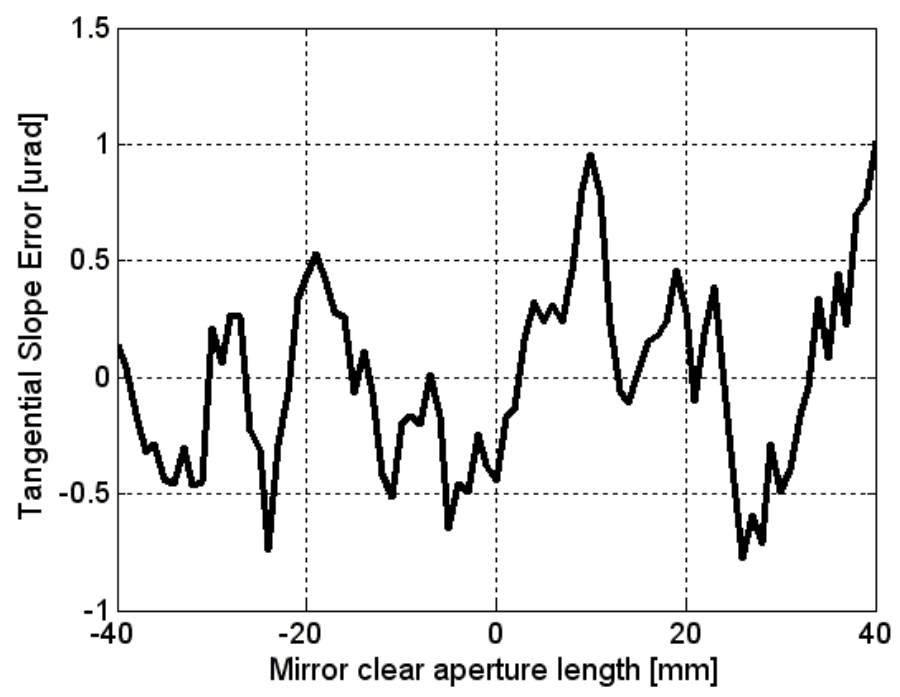

Figure 3: The residual slope error of the mirror tangential slope after subtraction of the desired elliptical shape. 
Figure 3 shows the residual variation of the mirror tangential slope error prior to beamline installation. Rigid-body optimization of mirror displacement and tilt, however, must be performed in-situ.

From the measured data (Fig. 3), two sets of information can be obtained. First, there are the relatively large slope error features at the higher spatial frequencies: these are probably artifacts of the mirror polishing. It could partially be due to a contribution of the LTP systematic errors. Second, there is larger amplitude mirror slope variation seen at the lower spatial frequencies (figure error). Since the mirror is not being used at the original design specifications, we anticipate some residual low-frequency errors may remain. However, the alignment algorithm is able to remove shape errors up through 4th order. Cumulatively, the mirror slope errors result in aberrations, which blur the focal spot.

\section{In-situ shearing interferometry measurements}

After installation at the beamline, the test mirror pitch angle and position of the focal plane were optimally adjusted using scanning slits tests and an original method for optimal in-situ alignment, as described in Ref. 11. In the measurements described here, the mirror bending was not adjusted, remaining the same as it was set ex-situ, in the OML.

The scanning slit in-situ mirror slope measurements are performed using a YAG crystal placed near the focal plane, with a visible-light charged-coupled device (CCD) and a microscope objective to re-image the focused spot. The slits are used to isolate one small region of the mirror at a time. As the slits are scanned through the beam, the positions of the focused rays are evaluated in the YAG image, and the slope error can be determined from the motion of the beam centroid.

The algorithm of the in-situ alignment procedure is analogous of that of the procedure used for optimal setting of the mirror benders with the LTP (Sec. 4). Three scanning slit measurements with sequential change of the pitch angle and the focal position of a detector are performed. Based on the measurements, predictions for the optimal pitch angle and focal position are calculated using linear regression analysis. An optimal (converged) adjustment is obtained after two or three iterations [11].

Next we characterize the mirror's wavefront slope using lateral shearing interferometry. A binary grating structure with 1:1 line-to-space ratio is placed in the beam, downstream of the focus, and the various diffraction orders of the transmitted wavefront $W(x, y)$ overlap on an $\mathrm{x}$-ray CCD plane with small angular shear, producing an interference fringe pattern. The fringe pattern can be analyzed to reveal the wavefront slope in the direction of the shear [21]. As shown in Ref. 22, for coherently illuminated systems, lateral shearing interferometry offers a convenient wavefront slope measurement method with high sensitivity and the potential for high accuracy.

Figure 4 illustrates the in-situ shearing interferometer geometry. A relatively coarse grating period ( 4 to $6 \mu \mathrm{m}$ ) is used to minimize the angular shear and preserve the substantial overlap of the various diffraction orders in the CCD plane. (Larger shear gives greater sensitivity at the expense of a reduced overlap area.) For this purpose, an array of 200- $\mu$ m-long slits with a grating pitch values between 4 and 8- $\mu \mathrm{m}$ was created by electron-beam lithography. The grating array allows us to compare the performance of the different gratings for sensitivity and accuracy.

In the shearing experiments described in this paper, the soft x-ray wavelength $\lambda$ is $1 \mathrm{~nm}$; the KB mirror focus is $120 \mathrm{~mm}$ downstream of the mirror center; the used grating pitch $d$ is $6 \mu \mathrm{m}$; and 
the grating is placed close to the grating's first Talbot plane distance [21,22], $Z_{g} \approx d^{2} / \lambda=36 \mathrm{~mm}$ downstream of the mirror focus. Sharp fringes are obtained on the $\mathrm{x}$-ray CCD, which is $Z_{c c d}=1.5 \mathrm{~m}$ downstream of the mirror focus. When the grating is displaced by several $\mathrm{mm}$ from the optimal longitudinal position, in either direction, the fringe contrast and clarity decreases.

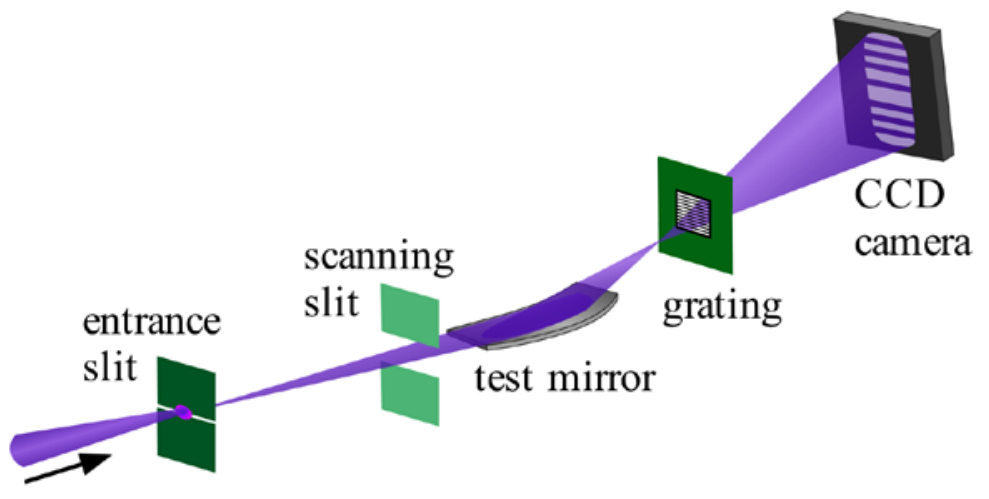

Figure 4: The geometry of in-situ shearing interferometry. Notice that due to the mirror's elliptical shape and the grazing incident geometry, there is a non-linear relationship between the beam position on the mirror surface and CCD plane.

Because of the mirror's elliptical shape and oblique angle of incidence, there is a non-linear relationship between the beam position on the mirror surface and its image position in the CCD plane. Therefore, uniform mirror surface sampling, such as is performed in the ex-situ slope profile measurements (as with the LTP-II), results in a non-uniform sampling in the CCD plane. Using commercial ray tracing software, ZEMAX ${ }^{\mathrm{TM}}$, we calculated the nonlinear mapping relationship from the mirror surface to the CCD plane with respect to the central ray: Fig. 5.

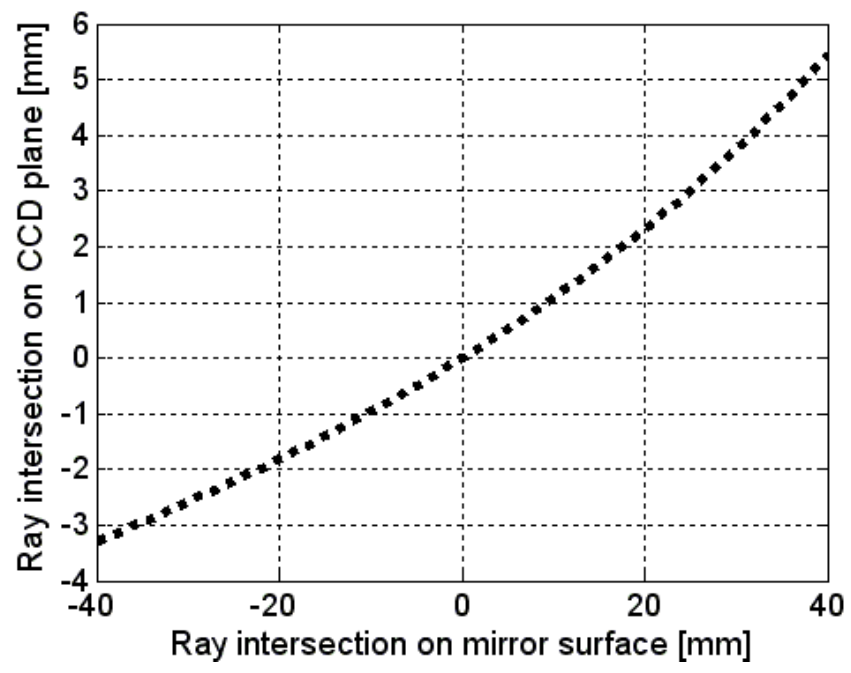

Figure 5: The non-linear relationship between a uniformly sampled mirror surface and the corresponding mapping in the CCD plane, with respect to the central ray.

Figure 6 shows typical shearing interferogram fringe patterns recorded by the CCD. 

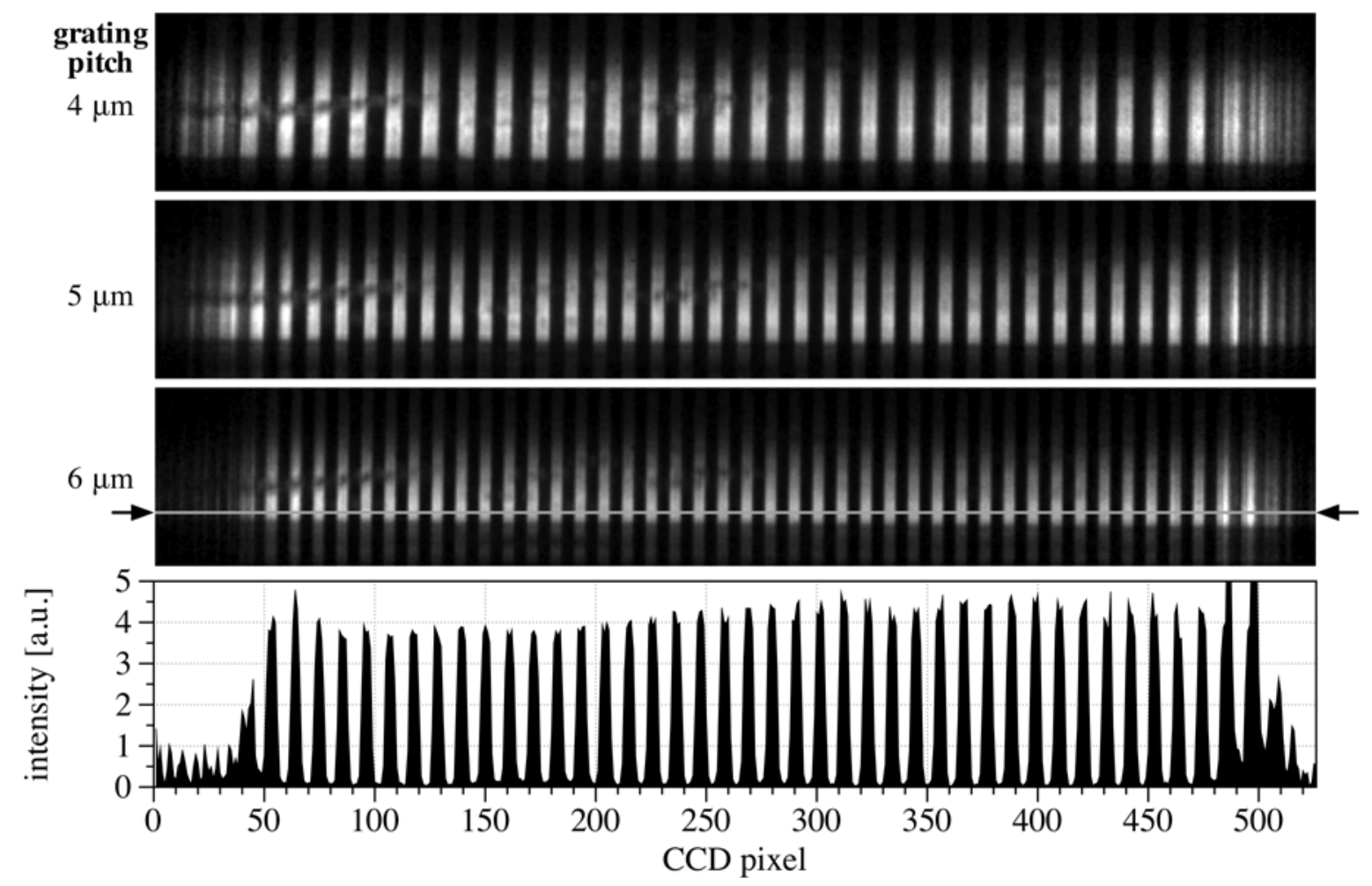

Figure 6: Shearing interferogram fringe patterns from three different gratings: 4, 5 and $6-\mu \mathrm{m}$ pitch. An intensity line-out from the 6- $\mu \mathrm{m}$ grating shows the high fringe contrast, with a nearly square intensity profile, achievable when the grating is placed close to the Talbot distance from focus. Some mirror surface contamination is visible on the left and in the center.

From geometrical optics, there is a relation between the wavefront slope error $\partial W(x, y) / \partial y$ ( $W(x, y)$ is the wavefront error) and the ray error $\varepsilon_{y}$ in the focal plane [21,23,24]:

$$
\partial W(x, y) / \partial y=\varepsilon_{y} / R,
$$

where $R$ is the local radius of curvature of the wavefront. From Eq. (1), the wavefront slope error is not a constant and will vary as the wavefront propagates. Indeed, the local radii of the wavefront exiting from the mirror surface is between $80 \mathrm{~mm}$ to $160 \mathrm{~mm}$ (Fig. 4), and the radii the wavefront at the CCD plane is $\sim 1500 \mathrm{~mm}$, the slope error on the CCD plane will be about 9.4 to 18.8 times smaller than that of the mirror surface.

Thus, the non-linear mapping and the propagation to the CCD requires that we stretch and scale the in-situ measured wavefront slope error from the CCD plane to match the mirror slope error trace measured with the LTP. Moreover, an appropriate correspondence should also account for the residual errors of the mirror alignment at the beamline, as well as for a possible error of the mirror bending due to a mechanical relaxation of the bender mechanisms. The LTP and the shearing measurements were performed 1.5 months apart. 


\section{Comparison of the in-situ and ex-situ test results}

The sharp fringes on the CCD plane, as shown in Fig. 6, they satisfy the fringe equation [21, p.126]:

$$
W D F=W(x, y+s / 2)-W(x, y-s / 2) \approx[\partial W(x, y) / \partial y]_{a v g} \cdot s=m \cdot \lambda,
$$

where WDF is the wavefront difference function; $s$ is the shearing distance, which equals $s=\lambda\left(Z_{c c d}-Z_{g}\right) / d ;[\partial W(x, y) / \partial y]_{\text {avg }}$ is the wavefront slope averaged over the shearing distance $s$; and $m$ is the fringe order number. Equation (2) can be re-written as

$$
[\partial W(x, y) / \partial y]_{a v g}=W D F / s .
$$

In our shearing data analysis, the Fourier transform method [25] is applied to obtain the wavefront difference function WDF from the individual fringe patterns, as a phase-map. (We note that phase-shifting analysis could also be used for the interferogram analysis provided a series of measurements with laterally shifted grating positions.) Equation (3) is applied to obtain the averaged wavefront slope error on the CCD plane. The linear slope term corresponds to the defocus caused by the grating's position downstream of the focus. We subtract this term [25] when investigating the wavefront slope errors.

To cross-check the in-situ and ex-situ metrology, we map the OML LTP-II measured mirror slope error trace onto the CCD plane by stretching the trace according to the calculated relationship (Fig. 5) and scaling by the varying magnification factors (Fig. 4). Figure 7 compares the result of the wavefront slope error measurements with the in-situ shearing interferometry discussed above (dash line), and the corresponding slope error trace obtained from the ex-situ LTP-II measurement with the bent mirror (solid line).

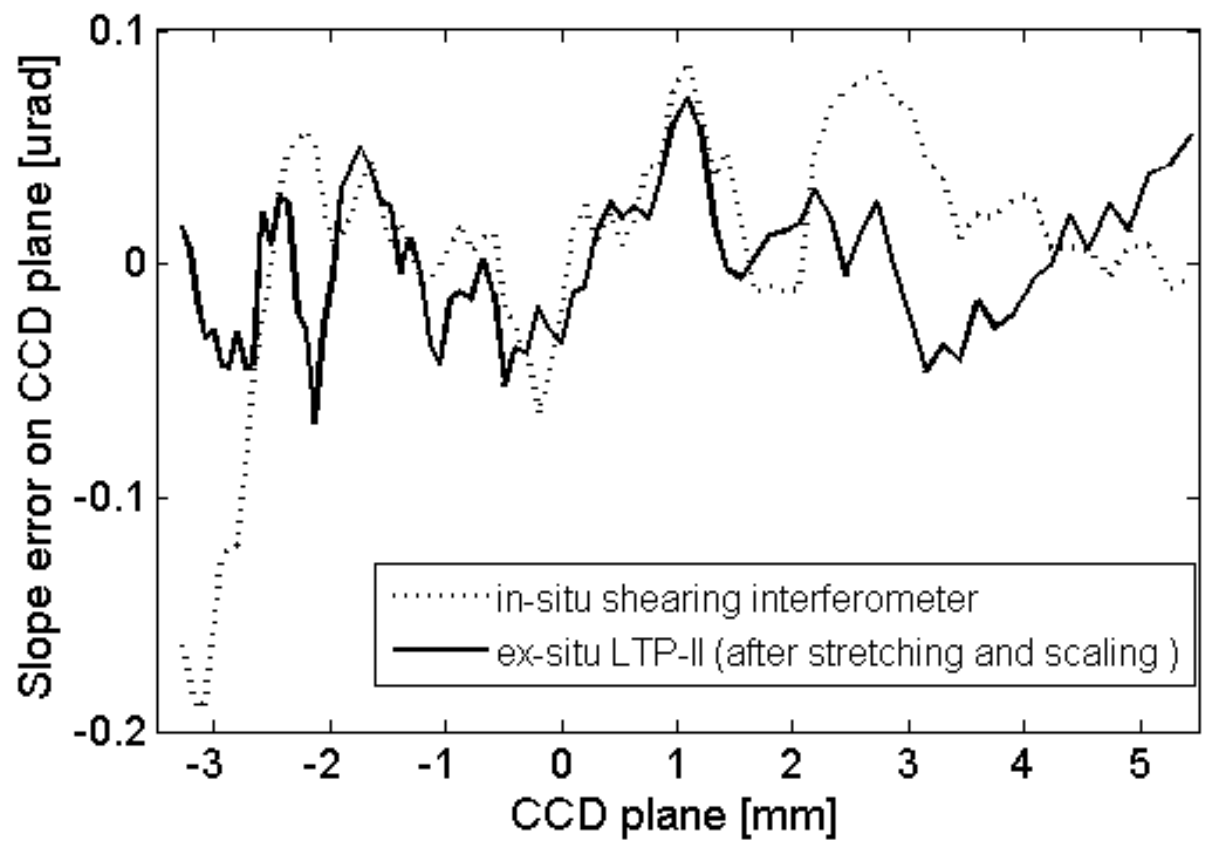

Figure 7: The measured wavefront slope by shearing interferometer (the dashed line) and the LTP measured slope error (the solid line). The LTP data are mapped onto the CCD plane by stretching and scaling as discussed in the text. 
The data in Fig. 7 suggest that the in-situ and ex-situ measured wavefront slopes are close in magnitude and strongly correlated. Indeed, the variance of each single measurement in Fig. 7 is larger than the variance of the difference of the measurements by a factor of approximately two.

We observe that the agreement is better around the mirror center. However, on the sides of the plot there is a noticeable discrepancy. The discrepancy may indicate the presence of a residual error of the mirror alignment at the beamline. There could also be a difference in the bending of the mirror due to a possible mechanical relaxation of the bending mechanism. We should also mention that the LTP calibration, performed with a diffraction grating, does not allow us to reliably account for the instrumental systematic error. The development of a more sophisticated calibration method based on the concept of a Universal Test Mirror, suggested in Ref. 26 [26], is in progress.

We recognize that the primary objective of the in-situ alignment is the optimized setting of the low-spatial frequency mirror shape, which can be controlled through bending [20]. Given the uncertainties in the mirror alignment between the two systems, we anticipate that the higher spatial frequency variations of the shearing interferometry and the LTP data should agree even better than the full-slope data shown in Fig. 7. Therefore, a comparison of higher spatialfrequency wavefront slope variations could provide a separate validation of performance of the performance of the in-situ metrology.

To isolate the higher-spatial-frequency variations of the mirror slope error traces, we fit and subtract aberration polynomials up to fourth-order. Figure 8 shows a comparison of the obtained higher spatial frequency variations of the wavefront slopes; the solid line is for the ex-situ LTP-II and the dashed line is for the in-situ shearing interferometry.

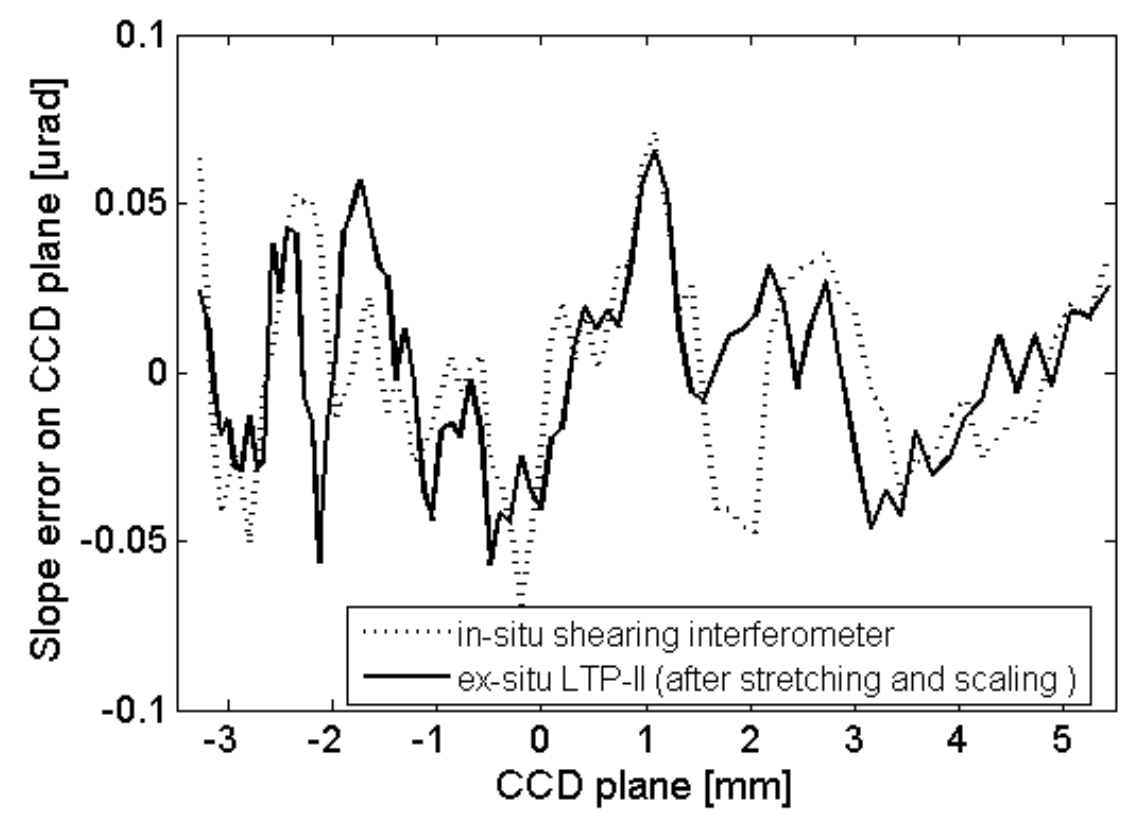

Figure 8: The higher spatial frequency variations of the slope error measured by the in-situ shearing interferometer (the dashed line) and the ex-situ LTP-II (the solid line), after subtracting the best-fit fourth order polynomials. The rms variation of the difference of both data corresponds to $\sim 0.20 \mu \mathrm{rad}$ (rms) slope difference on the mirror surface. 
Figure 8 shows the correlation between the higher spatial frequency components of the mirror slope error, measured in situ and ex situ. The (rms) variation of the data difference is $\sim 0.016$ urad on the CCD plane, which is $1.5 \mathrm{~m}$ downstream of the KB mirror focus. This variation corresponds to $\sim 0.20 \mu \mathrm{rad}$ (rms) slope difference on the mirror surface.

\section{Conclusions and Discussion}

At the ALS, a set of in-situ, at-wavelength metrologies for application with soft x-ray focusing optics are currently under development [10,11]. The first results of application of an in-situ shearing interferometry technique to characterize the surface quality of a bendable temperaturestabilized KB mirror have been reported and discussed. The details of the test mirror design, the ex-situ, optimal bender setting, and in-situ, optimal alignment at the beamline have been shown.

We have also reported a cross-check of the ex-situ LTP-II and in-situ shearing interferometry that became possible after a careful mapping the KB mirror slope error data measured with the ALS LTP-II to the CCD plane of the shearing interferometer. We have observed a good agreement of the data, especially around the mirror center. Even better correlation of the in-situ and ex-situ measurements has been found for the higher spatial frequency variations of the data. In this case, the possible spurious effects related to the LTP systematic error and a possible instability of the test mirror shape are significantly suppressed. The rms variation of the higher spatial frequency data difference has been found to be equivalent to approximately 0.2- $\mu$ rad (rms) slope variation of the surface slope trace as it is measured with the LTP-II. An additional investigation for the origin of the low spatial frequency discrepancy of the measurements is in progress.

We should also mention that the high performance of the ALS optical metrology and the developed in-situ alignment and characterization methods have lead to almost diffraction limited focusing (with the focus spot size of $\sim 120 \mathrm{~nm}$ ) with this test mirror [11].

\section{Acknowledgements}

The authors are grateful to Erik Anderson of CXRO for nanofabrication. The Advanced Light Source is supported by the Director, Office of Science, Office of Basic Energy Sciences, Material Science Division, of the U.S. Department of Energy under Contract No. DE-AC0205CH11231 at Lawrence Berkeley National Laboratory.

\section{Disclaimer}

This document was prepared as an account of work sponsored by the United States Government. While this document is believed to contain correct information, neither the United States Government nor any agency thereof, nor The Regents of the University of California, nor any of their employees, makes any warranty, express or implied, or assumes any legal responsibility for the accuracy, completeness, or usefulness of any information, apparatus, product, or process disclosed, or represents that its use would not infringe privately owned rights. Reference herein to any specific commercial product, process, or service by its trade name, trademark, manufacturer, or otherwise, does not necessarily constitute or imply its endorsement, recommendation, or favoring by the United States Government or any agency thereof, or The Regents of the University of California. The views and opinions of authors expressed herein do not necessarily state or reflect those of the United States Government or any agency thereof or The Regents of the University of California. 


\section{References}

1. R. D. Geckeler, "ESAD Shearing Deflectometry: Potentials for Synchrotron Beamline Metrology,” Proc. SPIE 6317, 63171H-1-13 (2006).

2. F. Siewert, T. Noll, T. Schlegel, T. Zeschke, and H. Lammert, “The Nanometer Optical Component Measuring Machine: a new Sub-nm Topography Measuring Device for X-ray Optics at BESSY,” AIP Conf. Proc. 705, 847-850 (2004).

3. V. V. Yashchuk, S. Barber, E. E. Domning, J. L. Kirschman, et al., "Sub-microradian Surface Slope Metrology with the ALS Developmental Long Trace Profiler,” Nucl. Instr. and Meth. A 616, 212-223 (2010).

4. S. Mourikis, W. Jark, E. E. Koch, V. Saile, "Surface temperature and distortion of optical elements exposed to high power synchrotron radiation beams," Rev. Sci. Instrum. 60 (7), 1474-8 (1989).

5. T. Warwick, S. Sharma, "Thermal effects and mirror surface figure requirements for a diagnostic beamline at the Advanced Light Source,” Nucl. Instr. and Meth. A 319, 185-7 (1992).

6. N. Kihara, K. Mashima, S. Miura, A. Miyaji, K. Wakamiya, S. Ichikawa, "Thermal and deformation analyses of side-cooled monochromator mirrors for the Spring-8/Figure-8 soft x-ray undulator,” J. Synchrotron Rad. 5, 811-3 (1998).

7. K. Freund, “Challenges for synchrotron x-ray optics,” Proc. SPIE 4782, 1-12 (2002).

8. S. Yuan, M. Church, V. V. Yashchuk, K. A. Goldberg, et al., "Elliptically Bent X-Ray Mirrors with Active Temperature Stabilization,” X-Ray Optics and Instrumentation 2010, 784732/1-9 (2010).

9. S. Yuan, V. V. Yashchuk, K. A. Goldberg, et al., "Surface Slope Metrology on Deformable Soft X-ray Mirrors,” AIP Conf. Proc. 1234, 789-792 (2010).

10. S. Yuan, K. Goldberg, V. V. Yashchuk, et al., "At-wavelength and optical metrology of bendable X-ray optics for nanofocusing at the ALS,” in Frontiers in Optics 2009, Laser Science XXV, Special Symposium on Optics for Imaging at the Nanoscale and Beyond (2009).

11. S. Yuan, K. A. Goldberg, V. V. Yashchuk, et al., "At-wavelength optical metrology development at the ALS,” Proc. SPIE 7801, 78010D/1-12 (2010).

12. M. Kunz, N. Tamura, K. Chen, A. A. MacDowel, et al., “A dedicated superbend x-ray micro-diffraction beamline for materials-, geo- and environmental sciences at the Advanced Light Source,” Rev. Sci. Instrum. 80 (8), 035108/1-10 (2009).

13. W. R. McKinney, S. C. Irick, J. L. Kirschman, et al., "New Procedure for the Adjustment of Elliptically Bent Mirrors with the Long Trace profiler,” Proc. SPIE 6704, 67040G (2007).

14. W. R. McKinney, S. C. Irick, J. L. Kirschman, A. A. MacDowell, A. Warwick, V. V. Yashchuk, "Optimal tuning and calibration of bendable mirrors with slope measuring profilers,” Opt. Eng. 48 (8), 083601 (2009).

15. J. L. Kirschman, E. E. Domning, W. R. McKinney, et al., "Performance of the upgraded LTP-II at the ALS Optical Metrology Laboratory,” Proc. SPIE 7077, 70770A-1-12 (2008). 
16. W. R. McKinney, M. Anders, S. K. Barber, et al., "Studies in Optimal Configuration of the LTP,” Proc. SPIE 7801, 7801-5/1-12 (2010).

17. S. K. Barber, G. Y. Morrison, V. V. Yashchuk, et al., "Developmental long trace profiler using optimally aligned mirror based pentaprism,” Proc. SPIE 7801, 7801-2/1-12 (2010).

18. V. V. Yashchuk, "Optimal Measurement Strategies for Effective Suppression of Drift Errors,” Rev. Sci. Instrum. 80, 115101-1-10 (2009).

19. V. V. Yashchuk, "Positioning errors of pencil-beam interferometers for long-trace profilers," Proc. SPIE 6317, 6317-10/1-12 (2006).

20. M. R. Howells, D. Cambie, R. M. Duarte, et al., "Theory and practice of elliptically bent Xray mirrors,” Opt. Eng. 39 (10), 2748-2762 (2000).

21. D. Malacara, “Optical shop testing”, 2nd ed. (Wiley, 1992).

22. P. P. Naulleau, K. A. Goldberg and J. Bokor, "Extreme ultraviolet carrier-frequency shearing interferometry of a lithographic four-mirror optical system,” J. Vac. Sci. \& Technol. B 18 (6), 2939-43 (2000).

23. S. Yuan, J. Sasian, "Aberrations of anamorphic optical systems. I: the first-order foundation and method for deriving the anamorphic primary aberration coefficients", Applied Optics, Vol. 48, Issue 13, pp. 2574-2584 (2009).

24. S. Yuan, J. Sasian, "Aberrations of Anamorphic Optical Systems II: The Primary Aberration Theory for Cylindrical Anamorphic Systems", Applied Optics, Vol. 48, Issue 15, pp. 28362841 (2009).

25. M. Takeda, H. Ina, and S. Kobayashi, "Fourier-transform method of fringe-pattern analysis for computer-based topography and interferometry,” J. Opt. Soc. Am. 72 (1), 156-160 (1982).

26. V. V. Yashchuk, W. R. McKinney, T. Warwick, et al., "Proposal for a Universal Test Mirror for Characterization of Slope Measuring Instruments,” Proc. SPIE 6704, 67040A (2007). 